\title{
Viabilidade de escleródios de Sclerotinia sclerotiorum e incidência de fungos antagonistas em solo de Cerrado
}

\author{
Leila de Castro Louback Ferraz ${ }^{1 *}$, Luiz Carlos Bhering Nasser ${ }^{2}$, Adalberto Corrêa Café-Filho ${ }^{3 *}$
}

${ }^{1}$ Rod. MG-424, Km 47 - Universidade Federal de São João del-Rei - CEP: 35.701-970, Sete Lagoas, MG; ${ }^{2}$ Embrapa Cerrados Caixa Postal: 08223 Planaltina, DF; ${ }^{3}$ Universidade de Brasília, Departamento de Fitopatologia, CEP 70.910-900 - Brasília, DF. * A primeira e o terceiro autores agradecem bolsa e apoio financeiro da CAPES e CNPq.

Autor para correspondência: Leila de Castro Louback Ferraz (louback@ufsj.edu.br)

Data de chegada: 01/06/2009. Aceito para publicação em: 10/10/2011.

\section{RESUMO}

Ferraz, L.C.L.; Nasser, L.C.B.; Café-Filho, A.C. Viabilidade de escleródios de Sclerotinia sclerotiorum e incidência de fungos antagonistas em solo de Cerrado. Summa Phytopathologica, v.37, n.4, p.208-210, 2011.

A viabilidade de escleródios de Sclerotinia sclerotiorum foi avaliada durante oito meses em três solos de Cerrado cultivados. Escleródios produzidos "in vitro", mantidos em invólucros de náilon perfurados, foram enterrados a $5 \mathrm{~cm}$ de profundidade, em solos previamente fumigados ou não fumigados com brometo de metila. Após 10 dias de incubação, os escleródios foram examinados quanto à viabilidade e a presença de fungos antagônicos. A viabilidade foi estimada através do número de escleródios germinados 7 dias após plaqueamento em meio semi seletivo Neon-S. A viabilidade dos escleródios variou com o solo de Cerrado. Escleródios incubados em solos não fumigados com brometo de metila apresentaram menor viabilidade e maior presença de fungos antagônicos, indicando que estes solos contêm elementos supressivos de origem biológica. A viabilidade dos escleródios foi relacionada negativamente com a população de alguns microorganismos de solo. Nos tratamentos de maior incidência de Trichoderma spp. observou-se menor viabilidade de escleródios e solos fumigados suprimiram fortemente a ocorrência deste antagonista.

Palavras-chave adicionais: Supressividade do solo, Fumigação, Trichoderma, Gliocladium

\section{ABSTRACT}

Ferraz, L.C.L.; Nasser, L.C.B.; Café-Filho, A.C. Viability of sclerotia of Sclerotinia sclerotiorum and incidence of antagonistic fungi in Cerrado soils. Summa Phytopathologica, v.37, n.4, p.208-210, 2011.

The viability of sclerotia of Sclerotinia sclerotiorum was assessed for eight months in three cultivated Cerrado soils. Sclerotia produced in vitro were placed in nylon mesh bags and buried $5 \mathrm{~cm}$ deep in soils either previously treated or untreated with methyl bromide. After 10 days of incubation, sclerotia were retrieved and were examined for viability and presence of antagonistic fungi. Viability was assessed based on the number of sclerotia germinated after a 7-day incubation period on semi-selective Neon-S medium. Viability of the sclerotia varied among Cerrado soils. Sclerotia from soils not treated with methyl bromide were less viable and were often contaminated by antagonistic fungi, indicating the presence of suppressive elements of biological origin in the untreated Cerrado soils. Sclerotium viability was negatively related to the population of some soil microorganisms. Treatments with higher incidences of Trichoderma spp. also presented lower sclerotium viability, and fumigation strongly suppressed this antagonist.

Keywords: Soil suppressiveness, Soil fumigation, Trichoderma, Gliocladium

Os registros de tempo de sobrevivência de escleródios de Sclerotinia sclerotiorum (Lib.) de Bary no solo variam bastante na literatura, dependendo de fatores tais como a estrutura física do escleródio, adubação, aplicação de fungicidas, umidade, matéria orgânica no solo e presença de parasitas e predadores $(1,3,6)$. O enterrio dos escleródios é geralmente recomendado para reduzir sua viabilidade, enquanto que o nível de matéria orgânica no solo pode tanto comprometer a viabilidade $(3,5)$, quanto favorecer o aumento do número de escleródios de $S$. sclerotiorum (2), assim como a germinação carpogênica (3). Mais de 30 espécies de fungos e bactérias foram relatadas como antagonistas ou agentes potenciais de controle biológico de Sclerotinia spp. Os gêneros de fungos mais frequentemente citados são Gliocladium, Coniothyrium, Trichoderma e Paecilomyces (9).

Este trabalho relata a dinâmica de alterações de viabilidade de escleródios de S. sclerotiorum em solos de Cerrado cultivados, durante um período de oito meses, descrevendo também a presença de fungos antagônicos associados aos escleródios.

Os ensaios foram conduzidos em três solos de Cerrado do Distrito Federal, com diferentes históricos de cultivo, no período entre 15 de maio de 1995 a 10 de janeiro de 1996 (outono ao verão do ano seguinte). Escleródios do isolado UnB-916 foram cultivados na ausência de luz em meio BDA por sete dias a $18^{\circ} \mathrm{C}$. A seguir, discos de micélio foram transferidos para cerca de $12 \mathrm{~kg}$ de meio cenoura + fubá (10\%) e incubados por 30 dias, no escuro a $20^{\circ} \mathrm{C}$. Depois de retirados do meio de cenoura, cinco gramas destes escleródios (40 a 60 escleródios) foram enterrados a cinco $\mathrm{cm}$ de profundidade, em invólucros de náilon perfurados para facilitar sua recuperação. Nos locais denominados "Planaltina", "PAD 1" e "PAD 2", foi testada a hipótese de 
supressividade natural do solo.

As áreas denominadas "PAD/DF 1" e "PAD/DF 2" corresponderam a duas metades em quadrantes opostos de um único pivô central cultivado com feijão na época do inverno, e milho no verão, em propriedade localizada no Plano de Assentamento Dirigido do Distrito Federal - PAD/DF. Ambas as áreas foram cultivadas em sistema de cultivo direto sobre palhada de trigo, entre 1991 e 1995 (PAD/DF 2) e entre 1994 e 1995 (PAD/DF 1). A área PAD/DF 1 recebeu 5 ton/ha de cama de frango em 1994. Os solos dos três locais foram classificados como latossolos argilosos (41 a 50\% de argila), com $\mathrm{pH}$ entre 5,4 e 6,2 .

As parcelas experimentais foram delimitadas em uma área de quatro $\mathrm{m}^{2}$. Cinco dias antes do enterrio dos escleródios foi realizada a fumigação do solo com brometo de metila, em metade das parcelas em cada local. As coletas dos escleródios foram realizadas aos 15, 30, 60, 90, 105, 148, 192, 240 dias após o enterrio. Foram coletados cerca de 40 escleródios por tratamento, em cada data de coleta. Depois de coletados, os escleródios foram lavados em água corrente e desinfestados superficialmente, após secos e plaqueados com cinco escleródios por placa em meio semi-seletivo Neon-S (7), a $18^{\circ} \mathrm{C}$, por 7 dias, no escuro, seguidos por 3 dias em fotoperíodo de $12 \mathrm{~h}$. Foram utilizadas placas de Petri, com meio Neon-S, contendo 5 escleródios por placa. As avaliações foram feitas a cada $24 \mathrm{~h}$ durante sete dias, observando-se a formação de um halo amarelado em torno dos escleródios (consequiência do processo de germinação de $S$. sclerotiorum), o que foi considerado indicativo da viabilidade de cada unidade de escleródio (7). Completados 10 dias de incubação (com 3 dias adicionais em fotoperíodo de $12 \mathrm{~h}$, à $22^{\circ} \mathrm{C}$ ), os escleródios foram avaliados quanto à presença de fungos, os quais foram classificados ao nível de gênero. O delineamento experimental utilizado foi inteiramente casualizado com esquema fatorial 7 × 3 × 2, com oito repetições, respectivamente com a ordem dos fatores de: datas de coleta, local de enterrio dos escleródios, e do tratamento prévio do solo com brometo de metila. Cada repetição
Tabela 1: Viabilidade média dos escleródios e Incidência de escleródios contaminados após o enterrio em três solos de Cerrado (Planaltina, PAD/ $\mathrm{DF} 1$ e PAD/DF 2) com tratamento prévio de não fumigação ou previamente fumigados com brometo de metila.

\begin{tabular}{lcc}
\hline Solo de Cerrado & \multicolumn{2}{c}{ Viabilidade (Germinação em meio Neon-S) } \\
\cline { 2 - 3 } & Não fumigado & Fumigado \\
\hline Planaltina & 40,9 a A & 86,4 a B \\
PAD/DF 1 & $52,2 \quad$ b A & $78,5 \quad$ b B \\
PAD/DF 2 & $58,1 \quad$ c A & $73,3 \quad$ b B \\
\hline Média & 50,4 & 79,4 \\
\hline & Incidência de Escleródios Contaminados \\
\cline { 2 - 3 } & Não fumigado & Fumigado \\
\hline Planaltina & 11,7 a A & 13,5 a A \\
PAD/DF 1 & 14,5 a A & $5,3 \quad$ b B \\
PAD/DF 2 & 15,9 a A & 20,1 a A \\
\hline Média & 14,0 & 13,0 \\
\hline
\end{tabular}

* Letras maiúsculas indicam comparação das médias dentro da mesma linha e letras minúsculas indicam comparação na mesma coluna, segundo teste de Tukey $(\mathrm{p} \leq 0,05)$.

consistiu de uma placa com meio Neon-S com cinco escleródios.

Foram encontrados escleródios viáveis até a última coleta, oito meses após o enterrio, em todos os solos e tratamentos. Sobrevivência de escleródios de $S$. sclerotiorum em solo natural após oito meses está de acordo com a literatura (4). Na maioria das vezes, os escleródios que não germinaram após sete dias de incubação também estavam associados a fungos antagônicos aos 10 dias de incubação, e observouse uma correlação significativa entre o número de escleródios inviáveis e os escleródios contaminados (calculada pela correlação de Pearson). A viabilidade dos escleródios foi menor nas coletas aos 30, 60 e 105 dias, enquanto a presença de contaminantes fúngicos nos escleródios
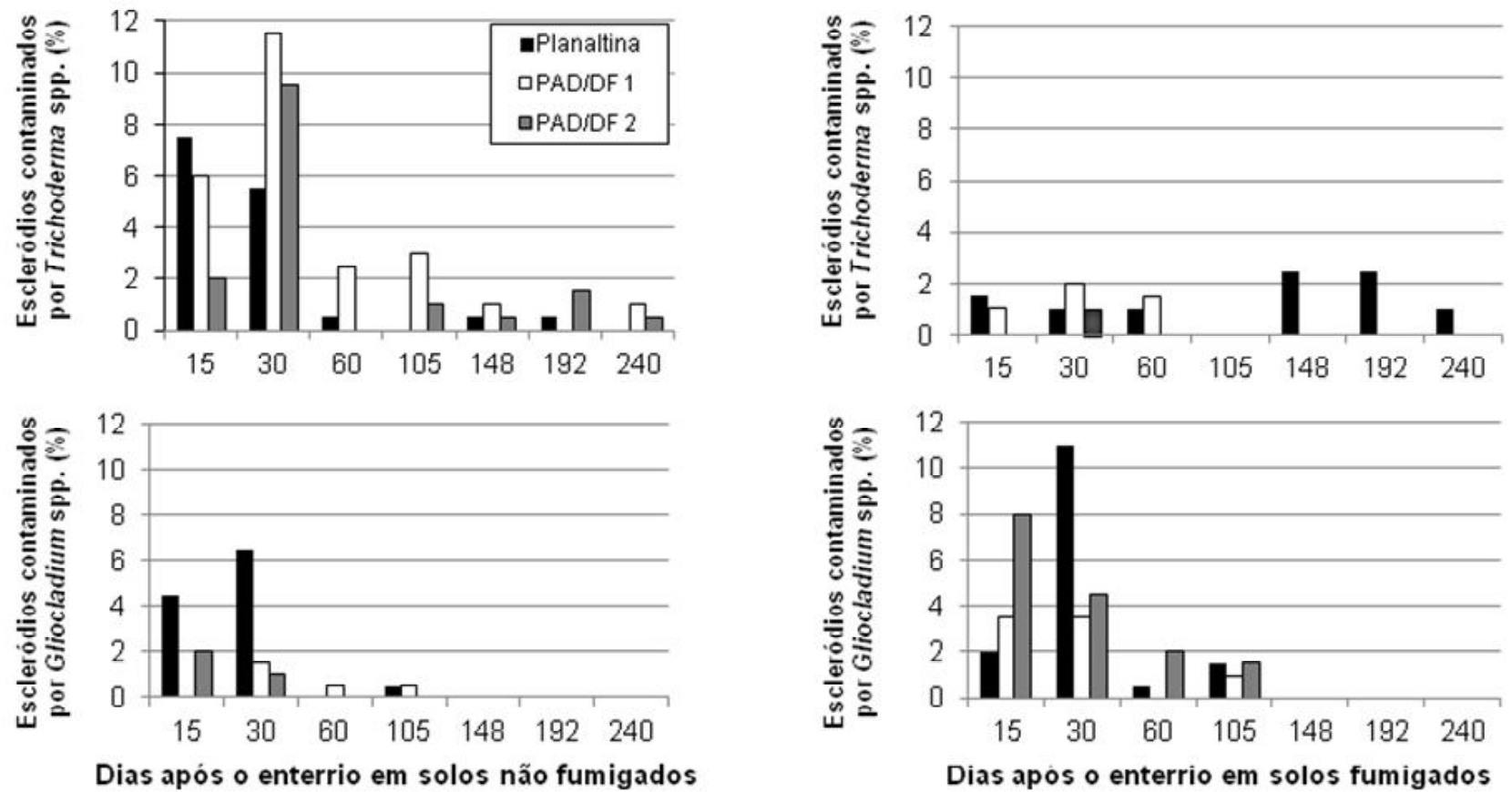

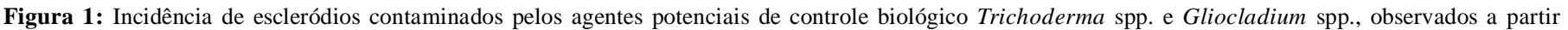
de escleródios após 10 dias incubação em meio Neon-S, proveniente de três solos de Cerrado (Planaltina, PAD/DF 1 e PAD/DF 2) sem tratamento prévio (solos não fumigados, esquerda) ou com tratamento prévio com brometo de metila (solos fumigados, direita), em dias após o enterrio dos escleródios. 
foi máxima aos 30 dias de enterrio. Nas duas últimas coletas (192 e 240 dias), observou-se que alguns escleródios recuperados dos solos se encontravam degradados. Isto pode estar relacionado ao início do período de chuvas e à presença de plantas invasoras, as quais podem estimular a germinação dos escleródios através de exsudados das raízes $(4,8)$. Por outro lado, a germinação dos escleródios também pareceu estimular a atuação de fungos antagônicos, conforme anteriormente relatado (4).

A dinâmica de viabilidade dos escleródios foi fortemente influenciada pelo solo das três localidades de Cerrado avaliadas. Em solos não tratados, a viabilidade dos escleródios foi menor em Planaltina, seguida de PAD/DF 1 e de PAD/DF 2, enquanto que a incidência de agentes contaminantes fúngicos nos escleródios não diferiu entre os três solos de Cerrado (Tabela 1). Quando os solos foram fumigados com brometo de metila a viabilidade dos escleródios aumentou em todos os locais estudados, mas a incidência de contaminantes foi pouco afetada (Tabela 1).

Em todos os locais foi observada maior incidência de fungos antagônicos aos 30 dias (Figura 1). Este efeito pode ser explicado por terem sido utilizados escleródios jovens, cultivados "in vitro", supostamente mais suscetíveis à colonização por fungos antagônicos, em contraste aos escleródios mais maduros, que completaram a maturação do anel exterior. Com o passar do tempo de incubação no solo, os escleródios produzidos "in vitro" tornam-se menos suscetíveis à colonização por antagonistas, ao completarem a formação deste anel de proteção. Esta observação está de acordo com Mitchel \& Wheeler (6), que observaram perda de viabilidade mais rápida de escleródios produzidos "in vitro" em comparação com aqueles coletados em campo.

Os resultados qualitativos de incidência de fungos antagônicos nos escleródios demonstraram a predominância de Gliocladium e Trichoderma em todos os solos, principalmente em "Planaltina", que apresentou a maior porcentagem de Trichoderma spp. aos 15 dias e de Gliocladium spp. aos 15 e 30 dias (Figura 1). Em alguns tratamentos também verificou-se a presença de Penicillium, Verticillium, Fusarium, Rhizoctonia, Aspergillus, Cladosporium e do nematóide micófago Aphelenchoides sp. Nos três solos estudados, a fumigação reduziu drasticamente a incidência de Trichoderma, permanecendo alta a incidência de Gliocladium, indicando que o fumigante desfavoreceu seletivamente a população natural de Trichoderma e favoreceu a de Gliocladium (Figura 1). Um resultado semelhante quanto a ocorrência de patógenos de solo foi apresentado por Venâncio et al. (10) que observaram um menor presença fungos do solo, em termos quantitativos, provenientes de solo fumigado, em até $25 \mathrm{~cm}$ de profundidade. Os resultados obtidos neste estudo demonstram diferenças entre o comportamento dos agentes potenciais de controle biológico em serem suprimidos ou não devido à fumigação prévia deste solo, como no caso dos gêneros Trichoderma e de Gliocladium, que apresentaram comportamentos distintos.

Escleródios de $S$. sclerotiorum produzidos "in vitro" permaneceram viáveis por um período de 240 dias (oito meses) em solos de Cerrado. Os resultados sugerem que todos os solos estudados, especialmente "Planaltina", contêm fatores supressivos de origem biológica. Também confirmaram numerosas observações anteriores que indicam que os gêneros Trichoderma e Gliocladium estão envolvidos no processo de supressividade. Estes dados reforçam a importância do potencial da microbiota natural dos solos de Cerrado na redução da viabilidade de S. sclerotiorum, principalmente em áreas com histórico de plantio direto, onde o mofo-branco é uma das doenças mais importantes dos cultivos de leguminosas no inverno.

\section{REFERÊNCIAS BIBLIOGRÁFICAS}

1. Clarkson, J.P.; Phelps, K.; Whipps, J.M.; Young, C.S.; Smith, J.A.; Watling, M. Forecasting Sclerotinia disease on lettuce: Toward developing a prediction model for carpogenic germination of sclerotia. Phytopathology, St. Paul, v.94, no. 3, p.268-279, 2004.

2. Durynina, E.P.; Efimenko, N.M.; Vostrikova, N.P. Development of Sclerotinia sclerotiorum (Lib.) de Bary during intensive fertilization in agroecosystems. Mikologia i Fitopatologiya, Saint Petersburg, v.23, p. 559-566, 1989.

3. Ferraz, L.C.L.; Café-Filho, A.C.; Nasser, L.C.B.; Azevedo, J. Effects of soil moisture, organic matter and grass mulching on the carpogenic germination of sclerotia and infection of bean by Sclerotinia sclerotiorum. Plant Pathology, Bognor Regis, v. 48, p.77-82, 1999.

4. Gasporotto, L.; Chaves, G.M. \& Condé, A.R. Sobrevivência de Sclerotinia sclerotiorum em solos cultivados com gramíneas. Fitopatologia Brasileira, Brasília, v. 7, p.223-232, 1982.

5. Mineev, V.G.; Durynina, E.P. Soil-agrochemical aspects of sunflower resistance to white rot (causal organism Sclerotinia sclerotiorum). Agrokhimiya, Moscow, v. 12, p.57-67, 1992.

6. Mitchel, S.J.; Wheller, B.E.J. Factors affecting the production of apothecia and longevity of sclerotia of Sclerotinia sclerotiorum. Plant Pathology, Bognor Regis, v. 39, p.70-76, 1990.

7. Napoleão, R.; Nasser, L.C.B.; Lopes, C.A.; Café-Filho, A.C. Neon$\mathrm{S}$, novo meio para detecção de Sclerotinia sclerotiorum em sementes. Summa Phytopathologica, Botucatu, v.32, n.2, pp.180$182,2006$.

8. Rimmer, S.R.; Menezes, J.G. Influence of host seedling exudates on germination of sclerotia of Sclerotinia sclerotiorum. Phytopathology, St. Paul, v. 73, p. 814. (Resumo).

9. Whipps, J.M.; Budge, S.P. Screening for sclerotial mycoparasites of Sclerotinia sclerotiorum. Mycological Research, Amsterdam, v. 94, p.607-612, 1990.

10. Venâncio, W.S.; Bueno, C.J.,; Souza, N.L. Efeito da solarização e do brometo de metila sobre a comunidade de fungos do solo. Summa phytopathologica, Botucatu, v.32, n.2, p. 183-185. 2006. 